\title{
Static Design of Spin Transfer Torques Magnetic Look Up Tables for ASIC Designs
}

\author{
Aliyar Attaran \\ School of Engineering, San Francisco \\ State University, San Francisco, CA \\ aattaran@mail.sfsu.edu
}

\author{
Tyler David Sheaves \\ School of Engineering, San Francisco \\ State University, San Francisco, CA \\ tsheaves@mail.sfsu.edu \\ Hamid Mahmoodi \\ School of Engineering, San Francisco \\ State University, San Francisco, CA \\ mahmoodi@sfsu.edu
}

\author{
Praveen Kumar Mugula \\ School of Engineering, San Francisco \\ State University, San Francisco, CA \\ pmugula@mail.sfsu.edu
}

\begin{abstract}
In this paper, we propose a static approach to the design of Spin Transfer Torque Look Up Tables (STT-LUT) for integration in ASIC and investigate the sensing reliability in the proposed design in detail. The proposed design style utilizes STT-Latches that their sensing reliability is key in determining the overall reliability of the proposed static STT-LUT. The simulation results in a $10 \mathrm{~nm}$ FinFET CMOS technology shows that the proposed static STTLUT design exhibits up to $26 \%$ read delay reduction compared to the best dynamic STT-LUT design, and more than $2.5 \mathrm{X}$ reduction in sensing failure rate.
\end{abstract}

\section{CCS CONCEPTS}

- Hardware $\rightarrow$ Integrated circuits; Hardware $\rightarrow$ Logic circuits; Hardware $\rightarrow$ Very large scale integration design; Hardware $\rightarrow$ Full-custom circuits; Hardware $\rightarrow$ Reconfigurable logic and FPGAs; Hardware $\rightarrow$ Application-specific VLSI designs; Hardware $\rightarrow$ Standard cell libraries

\section{KEYWORDS}

Look up the table, low power, a magnetic tunnel junction, reconfigurable logic, spin transfer torque

\section{INTRODUCTION}

Spin Transfer Torque Magnetic Memory (STT-MRAM) is a promising technology for non-volatile storage in which the information is stored in the form of magnetic orientation of a Magnetic Tunnel Junction (MTJ) rather than electric charge.

\footnotetext{
Permission to make digital or hard copies of all or part of this work for personal or classroom use is granted without fee provided that copies are not made or distributed for profit or commercial advantage and that copies bear this notice and the full citation on the first page. Copyrights for components of this work owned by others than ACM must be honored. Abstracting with credit is permitted. To copy otherwise, or republish, to post on servers or to redistribute to lists, requires prior specific permission and/or a fee. Request permissions from

Permissions@acm.org.

GLSVLSI '18, May 16-18, 2018, Chicago, IL, USA

(C) 2018 Association for Computing Machinery.

ACM ISBN 978-1-4503-5724-1/18/05 . \$15.00

https://doi.org/10.1145/3194554.319465
}

Besides memory applications, this technology is promising for CMOS compatible non-volatile reconfigurable logic design. Given the relatively high power and long delay associated with changing the magnetic state of an MTJ [1], the most efficient method of designing reconfigurable logic using MTJs is the Look-Up-Table (LUT) based approach. In such STT-LUTs, the write to MTJs occurs only during the configuration phase, while for the normal mode of the operation, the MTJs are only read from. Most existing STT-LUT designs are dynamic because of using a shared precharge sense amplifier to read the state of the selected MTJ. Dynamic STT-LUTs are challenging to use along with static combinational logic circuits and are not supported by automated design flow tools. Hence, we propose a static LUT design, in which MTJ-based precharge sense amplifier (PCSA) latches are used as storage cells. The basic programmable logic element is a look-up table that utilizes MTJs for storage (STT-LUT) [2] [3].

Existing designs of STT-LUTs utilize a shared dynamic precharge sense amplifier to read the state of the MTJ selected by the inputs. The dynamic design of the shared sense amplifier makes the entire STT-LUT a dynamic design. While dynamic designs have the advantage of less area using a shared sense amplifier, they suffer from long read delay, less reliability, and difficulty in integration with static logic and lack of support by automated design flow tools. The remainder of the paper is organized as follows. Section 2 offers a background on STT-LUT design and an overview of existing STT-LUT designs. Section 3 presents the proposed static approach to the design of STT-LUTs. Section 4 presents power, performance, and robustness comparisons of the proposed and conventional STT-LUTs. Section 5 described the integration of the proposed STT-LUT in ASIC designs. Finally, conclusions are drawn in Section 6.

\section{EXISTING STT-LUT DESIGNS: DYNAMIC}

In the existing dynamic approach, in n-input STT-LUT requires $2^{\text {n }}$ MTJs that store the binary states. A CMOS selection tree (i.e. multiplexer) select a unique MTJ to be biased for reading and a CMOS sense amplifier for producing full swing output voltage. In 
the write mode, the data input is written to a unique MTJ. The challenging aspect of STT-LUT design is reliable and power and performance efficient sensing of the MTJ resistive state and converting it to a binary voltage.

All existing STT-LUT designs use a variety of dynamic sensing schemes to update the sense amplifier output on a cycle by cycle basis. The SRAM based sensing scheme proposed in [3] uses an SRAM cell that is biased in the metastable condition in the initialization phase and then switches to the full-swing direction according to the resistance difference between the selected MTJ and a reference resistor. This design cannot be cascade because of the initial output voltage level being at around half the supply level (VDD) and hence distorting the sensing of the next stage. The other existing STT-LUT design is the Dynamic Current Mode (DCM) logic-based design [2]. This design uses the concept of the dynamic current mode logic [4], by utilizing a dynamic current source to reduce the current of the selection tree above it and hence to reduce the power consumption. However, by doing so, the read delay becomes very long. The value of the capacitance of the dynamic current source creates a trade-off between power and delay and if the capacitance is to be increased to achieve a competitive delay with other styles, its power consumption will become too high [4]. The Precharge Sense Amplifier (PCSA) STT-LUTs presented in [5] use MTJs in an array with single access transistors per MTJ, and hence relying on a separate decoder to provide the decoded MTJ select signals from primary LUT inputs. Separated Precharge Sensing Amplifier (SPCSA) is also presented in [5] as a more reliable style owing to higher voltage bias on the MTJs and a preamplification stage before the sense amplifier. However, the results in [6] show that the SPCSA adds too much area overhead in exchange for a faster operation. The Dynamic Dual Rail (DDR) STT-LUT style is presented in [6] and shown to be the most reliable and high-performance dynamic style for STT-LUTs. The DDR STT-LUT style shown in Fig. 1 is chosen as the representative of the state of the art dynamic STT-LUT style.

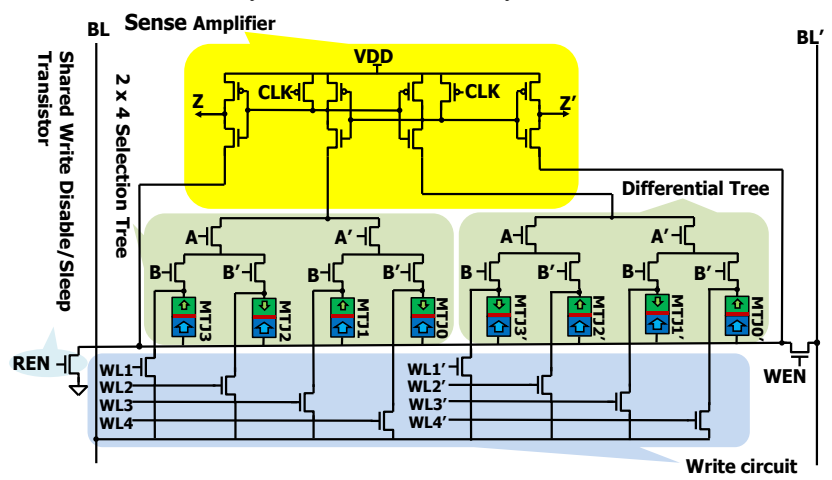

Figure 1. 2-input Dynamic Dual Rail (DDR) STT-LUT [6]

\section{PROPOSED STATIC STT-LUT DESIGN}

Fig. 2 shows the proposed Static STT-LUT style. By moving the multiplexer stage after the dynamic sense amplifier and using a static implementation of the multiplexer, the STT-LUT logic from input to output becomes static. In this scheme, each MTJ cell requires a unique dynamic latched sense amplifier. The sense amplifiers need to be enabled once at the beginning to have their outputs to be initialized according to the state of their MTJs. This needs to be done once on every power-on. The MTJs retain the content of the LUT in a non-volatile form. In this configuration, the MTJs are read only once and for the rest of the time in the active mode, the LUT read power and delay is determined by the static multiplexer. Moreover, by not reading from the MTJs repetitively as in the dynamic STT-LUT style, the stress is removed from the MTJs enhancing their life-time.

In fig. 3, The MTJ latch uses a pair of differentially programmed MTJs for non-volatile storage, a precharge sense amplifier for sensing the state of the MTJs, and three write driver scheme for parallel write to both MTJs simultaneously with each MTJ receiving full voltage swing, offering more write current. The Sense Enable (SE) signal must be low during the write operation, and the Write Enable (WE) signal must be low during the sensing operation. To avoid conflict of state between the precharge state of the sense amplifier (when $\mathrm{SE}=0$ ) and the state of the write driver outputs in the write mode, the precharge path to VDD is disconnected via the PMOS driven by the WE signal.

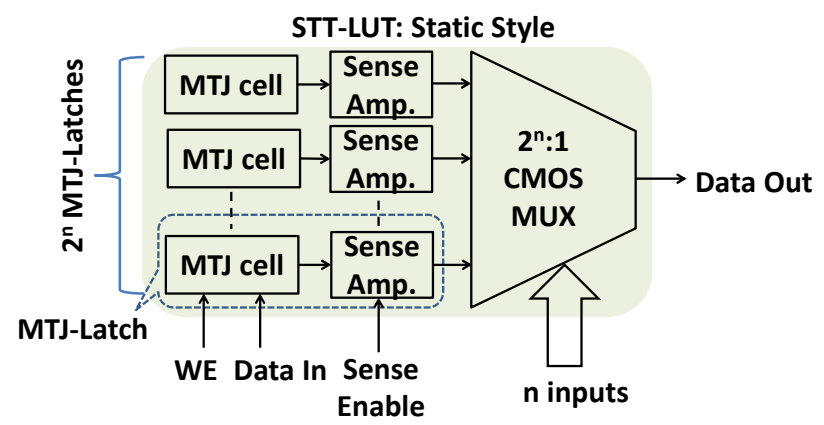

Figure 2. STT-LUT design concept: Proposed static style

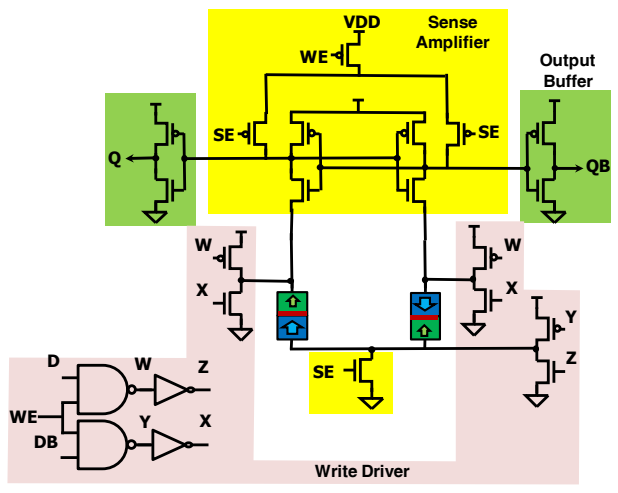

Figure 3. MTJ-Latch

The multiplexer of the LUT is a $2^{\text {n }}$ to $1\left(2^{\mathrm{n}}: 1\right)$ CMOS multiplexer (MUX) implemented in a static style. The power and performance of the proposed STT-LUT are determined by the multiplexer, but the reliability of its configuration is determined by the sensing reliability of the STT-latch itself.

\section{COMPARISONS}

\subsection{Simulation Setup}

The designs are simulated in a predictive $10 \mathrm{~nm}$ FinFET process [7] with a nominal supply voltage of $0.75 \mathrm{~V}$. To achieve a fair 
comparison, using the embedded Hspice optimizer, transistor sizes in both designs are optimized to minimize the Power-DelayProduct (PDP) under an equal constraint on the area which is $50 \%$ larger than the area of the bigger design when all transistors are minimum sized. Since the critical operation in programmable logic applications, where these LUTs are used, is the read mode, we simulated these designs in the read mode, where MTJs can be modeled as resistors. The MTJ resistance depends on the MTJ physical geometries and specifically the thickness of the insulator and the $2 \mathrm{D}$ cross-section area of the MTJ, and the voltage bias [8, 9]. The parallel state resistance $\left(\mathrm{R}_{\mathrm{P}}\right)$ can be modeled as $[8,9]$ :

$$
R_{P}=\frac{t_{o x}}{F \sqrt{\varphi} A} e^{\left(1.025 t_{o x} \sqrt{\varphi}\right)}
$$

where, $F$ and $\varphi$ are the tunneling conductivity and potential barrier height of the insulator, respectively. Considering a circular shape 2D area of radius $r$, area A is expressed as $A=\pi r^{2}$. The MTJ parallel resistance is estimated for the $16 \mathrm{~nm}$ process.

The resistance difference between the two MTJ states is quantified by the Tunneling Magneto Resistance (TMR) parameter [10]:

$$
T M R=\frac{R_{A P}-R_{P}}{R_{P}}
$$

A higher difference between $\mathrm{R}_{P}$ and $\mathrm{R}_{\mathrm{AP}}$ results in better read performance and reliability. Hence, for the STT-LUTs used in the reconfigurable logic application, higher TMR even if it is at the cost of higher written power and delay is desirable. Using crystalline $\mathrm{MgO}$ as the insulator, TMR up to 6 is reported [10]. In this study, we choose a TMR value of 4 .

\subsection{Results and Comparisons}

\subsubsection{Power, performance, and area comparisons}

In this section, the proposed static STT-LUTs from 2 to 6 fan-ins are characterized in terms of delay, active power, leakage power, PDP, area and sensing failure rate; and compared with the DDR STT-LUT. Unlike DDR, in static STT-LUT, the active power consumption is dependent on the switching activities. Therefore, for obtaining a more realistic evaluation of power comparison, the power of the STT-LUT designs was measured at $100 \%, 50 \%$ and $25 \%$ output switching activities. Fig. 4(a) shows the active power comparison which is dependent on output switching activity and Fig. 4(b) shows the leakage power which is measured in idle condition. From LUT2 to LUT6, 50\% to $43 \%$ of the total power is the leakage power and the rest is the switching power. The static LUTs show higher power consumption due to extra sense amplifies and hence more area. As shown in Fig. 4(a), the static LUTs active power in LUT2 is decreased by $25 \%$ and depending on fan-in and switching activity, the active power is increased by $1.5 \mathrm{X}$ to $10.9 \mathrm{X}$ from LUT3 or LUT6, as compared to the DDR LUTs. The Fig. 4(c) shows the delay comparison between static and DDR LUTs. The switching activity does not affect the delay of the circuit, and hence a single bar for static LUT is shown in Fig. 4(c). Compared to DDR, the delay of static LUT is improved by $14 \%, 17 \%, 26 \%, 15 \%$, and $19 \%$ for LUT2, LUT3, LUT4, LUT5, and LUT6, respectively. The reduction in delay is because in the static LUT the MTJ sensing delay is not in the critical path. The delay in the normal mode of operation is through a static multiplexer on the path from the select input to the output (Fig. 4). The MTJ sensing delay is not critical as in the proposed design, the MTJ sensing occurs only once at the system power up. After the initial MTJ sensing on power-up, the MTJ states are held by the latched outputs of the sense amplifiers.
It is also noticeable that in the proposed design, the stress on MTJs is removed in run-time because once the sense amplifier has finished sensing, the bias one the MTJs is reduced to zero volts, and hence eliminating any stress on the MTJs and resulting in longer life for the thin insulators of the MTJs. Low delay overhead and its static path from select inputs to output, makes the proposed LUT ideal for integration with static CMOS logic gates in hybrid custom/programmable hardware applications, where a design is implemented partially in LUTs and in custom static CMOS. Fig. 4(d) shows the total area of the static and DDR LUT designs. Except in low fan-in LUTS (LUT2 and LUT3), the area of the DDR LUT is lower due to the sharing of the sense amplifier. Using multiplexer (MUX) in static LUT and dedicated sense-amplifiers, makes the area of high fan-in LUTs larger compared to the DDR design. Fig. 4(d) shows that for LUT2, the static LUT shows a decrease in the area of $8 \%$. The area of the static LUT is increased by $36 \%$ and $19 \%, 24 \%$ and $14 \%$ in LUT3, LUT4, LUT5, and LUT6, respectively. Fig. 4(e) shows the measurement of PDP of static and DDR LUT styles. For static LUT with $25 \%$ switching activity, the PDP is decreased by $2 \mathrm{X}$ in LUT2 but increased by 1.64X, 2.31X, 4.90X, and 8.33X from LUT3 to LUT6.

\subsubsection{Robustness Comparisons}

There are two possible failures in the read mode: read sensing failure and read disturbance failure.
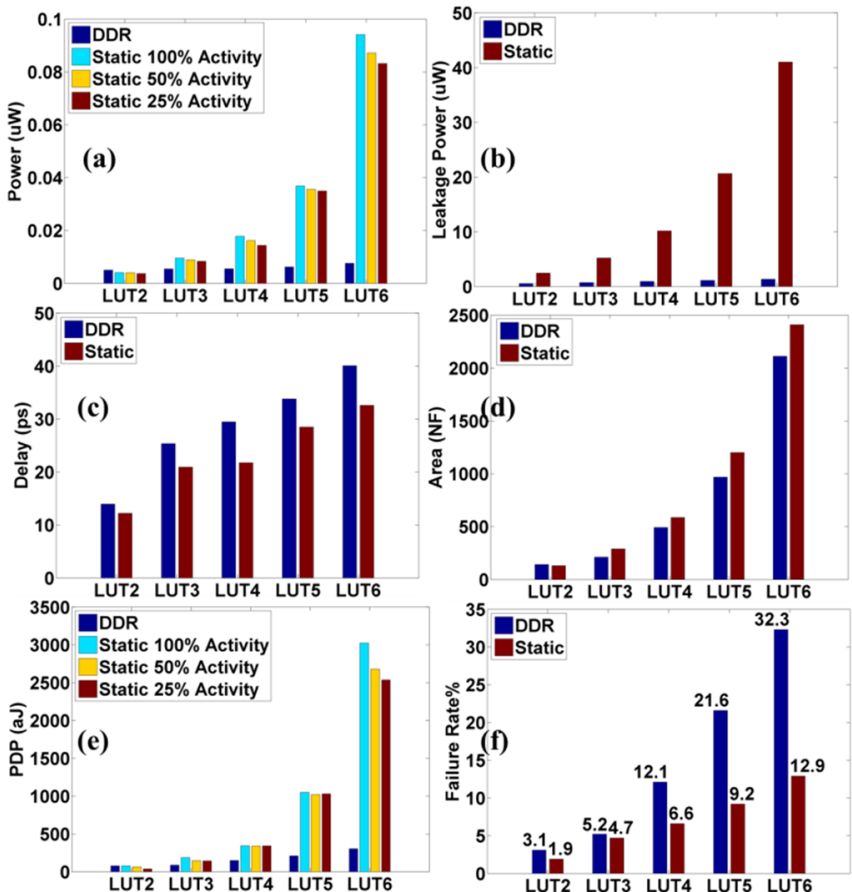

Figure 4. Performance comparisons: (a) Active Power (b) Leakage Power (c) Delay (d) Area (e) PDP (f) Failure rate

The read sensing failure is related to the incorrect sensing by the sense amplifier due to the presence of mismatch caused by CMOS and MTJ variations resulting in low sensing margin. The read disturbance failure is a sensing operation in which the sensing current passing through the MTJ exceeds the critical write current resulting in the flipping of the state of the MTJ (i.e. write operation). In STT-LUTs the read sensing failure is the dominant 
type because of the stack of transistors in the sensing path limits the read current. Hence, we focus on the read sensing failures. Random statistical variations are applied to MTJ parameters ( $t_{o x}$ and $r$ ) and threshold voltage $(d v t)$ and read sensing failure rates of the STT-LUTs are measured. The standard deviations of $t_{o x}$ and $r$ variations are set at $10 \%$ of their nominal values. From Fig. $4(\mathrm{f})$, it is very noticeable that the failure rate of static LUT is much lower than the DDR style. The low failure rate in static LUTs is due to lower number of transistors in the MTJ selection paths, which leads to increase in current differentials between the two differential MTJs in the latches and hence increase in the sensing margin in the sense amplifier. The failure rate reductions by static LUTs range from 1.6X to more than 2.5X depending on fan-in. As expected, the failure rate generally increases by fan-in for both LUT styles. However, the failures rates of the proposed design are dramatically lower even for high fan-in LUTs. For hardware reconfigurable applications, near zero failure rate is expected of LUTs. The conventional DDR design is not acceptable for such applications due to its very high failure rates. The proposed design offers very low failure rates and with the proper area, optimization can achieve zero failure rate, which of critical importance for programmable logic applications.

\section{INTEGRATION IN ASIC DESIGN}

For ASIC applications where an STT-LUT might be needed, it is desirable to be able to specify the STT-LUT at the RTL level and have the supporting models and libraries necessary for the ASIC automated design flow tools be able to insert the STT-LUT in the synthesized netlist and in the physical design stage. The proposed static STT-LUT has a multiplexer part which can be expressed in a synthesizable format at RTL. The other component is MTJ-Latch that is an analog mixed signal circuit that needs to be designed in a full custom fashion and delivered as a hard macro type standard cell. Offering MTJ latch as new standard cell and STT-LUTs as new macros for ASIC flows enables and improves design productivity for a range of new applications that can benefit from such programmable non-volatile elements. Such applications include automotive, security, hardware acceleration of computing, and field programmable logic, to name a few.

The MTJ latch is an analog mixed signal circuit as shown in Fig. 5. Therefore, for introducing it as a cell to the ASIC flow, it needs to be treated as a hard macro cell. Hard macros are typically larger blocks such as memory blocks for which custom layout is designed. Hard macros are typically placed in the floor planning step of the physical design along with other layout blocks that each might have been separately produced using the ASIC flow. A single bit MTJ latch is too small to be defined as a hard macro, and it is desired to be able to use it within a block at the RTL synthesis step. Hence, we propose to design the MTJ latch as a standard cell following the rules of standard cell design in terms of the layout width and height, placement of VDD and VSS layers, and the boundary layer. Fig. 5 shows the layout of the MTJ latch in the standard cell format. The MTJs are fabricated above the cell and Metal 2 pins are placed for connection to the MTJ layers. This layout is done after optimizing the MTJ latch for write and sense operations in the Synopsys 32/28nm PDK for which layout technology files are available.

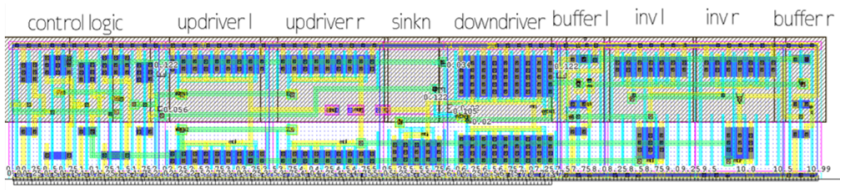

Figure 5. The layout of MTJ latch in standard cell format

\section{CONCLUSION}

In this paper, a static STT-LUT style is presented and compared with the state of the art dynamic counterpart. The existing STTLUTs that are dynamic cannot be used in ASIC designs that utilize static logic style gates (standard cells) due to the static to dynamic logic interface issue. The proposed design offers lower MTJ sensing failure rate and better speed. Moreover, dues to its static operation, the proposed design is much easier to integrate with the static CMOS logic and automated ASIC design flows. The comprehensive analysis of the proposed design and its comparison against the DDR LUT design shows that the proposed design superior in all aspects, except area and power at high fan-in LUTs. Its low failure rate even at high fan-ins makes the proposed design a reliable candidate for commercial hardware reconfiguration applications, in which near zero failure rate is expected of LUTs. An approach to integrating the proposed STT-LUT to the ASIC flow is also presented in which the MTJ latch is introduced as a hard macro standard cell, and the multiplexer portion of the STTLUT is represented in high-level synthesizable RTL code.

\section{ACKNOWLEDGMENTS}

This research is funded by the Defense Advanced Research Projects Agency (DARPA-AirForce, FA8650-15-C-7569).

\section{REFERENCES}

[1] M. Rasquinha, et al., "An energy efficient cache design using Spin Torque Transfer (STT) RAM," in Low-Power Electronics and Design (ISLPED), ACM/IEEE International Symposium on, 2010, pp. 389-394.

[2] D. Suzuki, et al., "Fabrication of a nonvolatile lookup-table circuit chip using magneto/semiconductor-hybrid structure for an immediate-power-up field programmable gate array," Symposium on VLSI Circuits, 2009, pp. 80-81.

[3] W. Zhao, et al. "Spin-transfer torque (STT)-MRAM--based runtime reconfiguration FPGA circuit," ACM Trans. Embed. Comput. Syst., vol. 9, pp. 1$16,2009$.

[4] M. W. Allam and M. I. Elmasry, "Dynamic current mode logic (DyCML): a new low-power high-performance logic style," IEEE Journal of Solid-State Circuits, vol. 36, pp. 550-558, 2001.

[5] E. Deng, et al. "Design Optimization and Analysis of Multicontext STT-MTJbased; CMOS Logic Circuits," IEEE Transactions on Nanotechnology, vol. 14, pp. 169-177, 2015.

[6] Ali Attaran, et al. "Dynamic Single and Dual Rail Spin Transfer Torque Look Up Tables with Enhanced Robustness under CMOS and MTJ Process Variations," ICCD, 2016.

[7] PTM. (2016). Available: http://www.eas.asu.edu/ ptm

[8] Y. Zhang, et al., "Compact Model of Subvolume MTJ and Its Design Application at Nanoscale Technology Nodes," IEEE Transactions on Electron Devices, vol. 62, pp. 2048-2055, 2015.

[9] Z. Xu, et al. "Compact modeling of STT-MTJ devices," Solid-State Electronics, vol. 102, pp. 76-81, 12// 2014.

[10] S. Ikeda, et al., "A perpendicular-anisotropy CoFeB-MgO magnetic tunnel junction," Nat Mater, vol. 9, pp. 721-724, 09//print 2010. 